\title{
Autoimmune disease: are vaccines a potential cause?
}

Currently available epidemiological evidence concerning the possible association between vaccines and autoimmune disease appears reassuring, say $\mathrm{Dr}$ David Wraith from the University of Bristol, UK, and colleagues.

To assess the relative risk of autoimmune diseases arising as a consequence of vaccination, Dr Wraith and colleagues examined the current understanding of how infections lead to autoimmune disease, noting that autoimmune diseases arise through complex interactions between genetic traits and environmental factors, and only in genetically predisposed individuals. They discuss a number of mechanisms by which infections may trigger autoimmune disease, including molecular mimicry and infection-induced autoimmunity with bystander activation. Dr Wraith and colleagues note that a number of fail-safe mechanisms exist to prevent immune response to infection from causing excessive tissue damage and thereby triggering autoimmune disease; these fail-safe mechanisms also apply to the host response to vaccination. However, they point out that "an effective vaccine should generate protective immunity while keeping to a minimum molecular mimicry and bystander activation".

Dr Wraith and colleagues note that only in rare cases has a link between vaccination and autoimmune disease been shown to exist, with large studies failing to find evidence for suggested links between hepatitis B vaccine and multiple sclerosis, and childhood vaccinations and type 1 diabetes mellitus. They point out that, when considering vaccine-associated risks, the risks associated with the corresponding natural infection also need to be considered. They add that, in vaccine development, "only a comprehensive and multidisciplinary strategy can help to reduce the risk that a new vaccine will induce autoimmune manifestations".

Wraith DC, et al. Vaccination and autoimmune disease: what is the evidence?

Lancet 362: 1659-1666, 15 Nov 2003 